\title{
Feasibility Study of Space Based Solar Power to Tethered Aerostat Systems
}

\author{
Stephen J. Blank \\ New York Institute of Technology \\ Northern Blvd. \\ Old Westbury, NY 11568 USA \\ 516-686-1302,sblank@nyit.edu \\ Stephen J. Leete \\ NASA Goddard Space Flight Center \\ Mission Systems Engineering Branch, Code 599 \\ Greenbelt, MD 20771 USA \\ 301-286-9093, stephen.j.leete@nasa.gov \\ Paul Jaffe \\ Naval Research Laboratory, Code 8243 \\ 4555 Overlook Ave SW \\ Washington, DC 20375, USA \\ (202) 767-6616, paul.jaffe@nrl.navy.mil
}

\begin{abstract}
The feasibility of two-stage Space-Based Solar Power to Tethered Aerostat to Earth (SSP-TA) system architectures that offer significant advantages over conventional single stage spaceto-earth architectures is being studied.

There have been many proposals for the transmission of solar power collected in space to the surface of the earth so that solar energy could provide a major part of the electric power requirements on earth. There are, however, serious difficulties in implementing the single stage space-based solar power systems that have been previously studied. These difficulties arise due to: i) the cost of transporting the components needed for the extremely large microwave transmit beaming aperture into space orbit, ii) the even larger collection apertures required on earth, iii) the potential radiation hazard to personnel and equipment on earth, and iv) a lack of flexibility in location of the collection station on the earth.

Two candidate system architectures are described here to overcome these difficulties. In both cases a two-stage space to tethered aerostat to earth transmission system (SSP-TA) is proposed. The use of high altitude tethered aerostats (or powered airships) avoids the effects of attenuation of EM energy propagating through the earth's lower atmosphere. This allows the use of beaming frequencies to be chosen from the range of high millimeter (THz) to near-infra-red (NIR) to the visible. This has the potential for: i) greatly reduced transportation costs to space, ii) much smaller receiver collection apertures and ground stations, iii) elimination of the potential radiation hazard to personnel and equipment on earth, and iv) ease in transportation and flexibility in location of the collection station on the earth. A preliminary comparison of system performance and efficiencies is presented.
\end{abstract}

\section{TABLE OF CONTENTS}

1. INTRODUCTION 1

2. CANDIDATE ARCHITECTURE SSP-TA-I .......................... 1

3. CANDIDATE ARCHITECTURE SSP-TA-II ........................ 3

4. PoWered Airship Alternative to the Tethered

Aerostat 4

5. COMPARISON OF THE ARCHITECTURES ......................... 4

6. CONCLUSIONS 5

978-1-4673-1813-6/13/\$31.00 C2013 IEEE
REFERENCES....................................................................6 6

BIOGRAPHY .................................................................6 6

1. INTRODUCTION

This is a report on a preliminary feasibility study of twostage Space Based Solar Power to Tethered Aerostat to Earth (SSP-TA) system architectures that offer significant advantages over conventional single-stage space-to-earth (SSP-SS) architectures.

There have been many proposals for the transmission of solar power collected in space to the surface of the earth so that solar energy could provide a major part of the electric power requirements on earth. An early description of such a system was Peter E. Glaser, December 25, 1973, "Method and Apparatus for Converting Solar Radiation to Electrical Power" [1]. In this patent, a single stage wireless power transmission system is proposed, Fig. 1. Following Glaser, W. C. Brown greatly contributed to the theory and design of such systems using microwave power beaming [2]. John Mankins [3] and others are currently studying space solar power. Al Globus [4] discusses possible solutions for an important problem that is common to all SSP systems, the need for large apertures in space to collect the desired amount of power.

There are, however, serious difficulties in implementing the single stage spaced-based solar power systems that have been proposed and are currently being studied. These difficulties arise due to: i) the cost of transporting the components needed for the extremely large microwave transmit beaming aperture into space orbit, ii) the even larger collection apertures required on earth, iii) the potential radiation hazard to personnel and equipment on Earth, and iv) a lack of flexibility in location of the collection station on the Earth.

Two candidate system architectures are described here to overcome these difficulties. In both cases a two-stage space to tethered aerostat to earth transmission system (SSP-TA) is proposed. 


\section{CANDIDATE ARChitecture SSP-TA-I}

2A. Description-We first discuss the two-stage system architecture shown in Figure 2 designated by SSP-TA-I [5,6]. Recently, a different group of investigators presented a study of a similar system, [7]. For this architecture, solar energy collected in space would be converted to collimated, monochromatic electromagnetic (EM) energy and beamed with high directivity towards the earth at a frequency chosen from the range of high millimeter (THz) to near-infra-red (NIR). This higher frequency range has previously received less attention due to the effects of high attenuation of EM energy propagating through the earth's lower atmosphere at these higher frequencies. But in the SSP-TA-I system there would be a second stage at an altitude of $6-9 \mathrm{~km}$ above the earth's surface where the beamed energy would be collected and converted to DC electric power. The DC electric power is sent

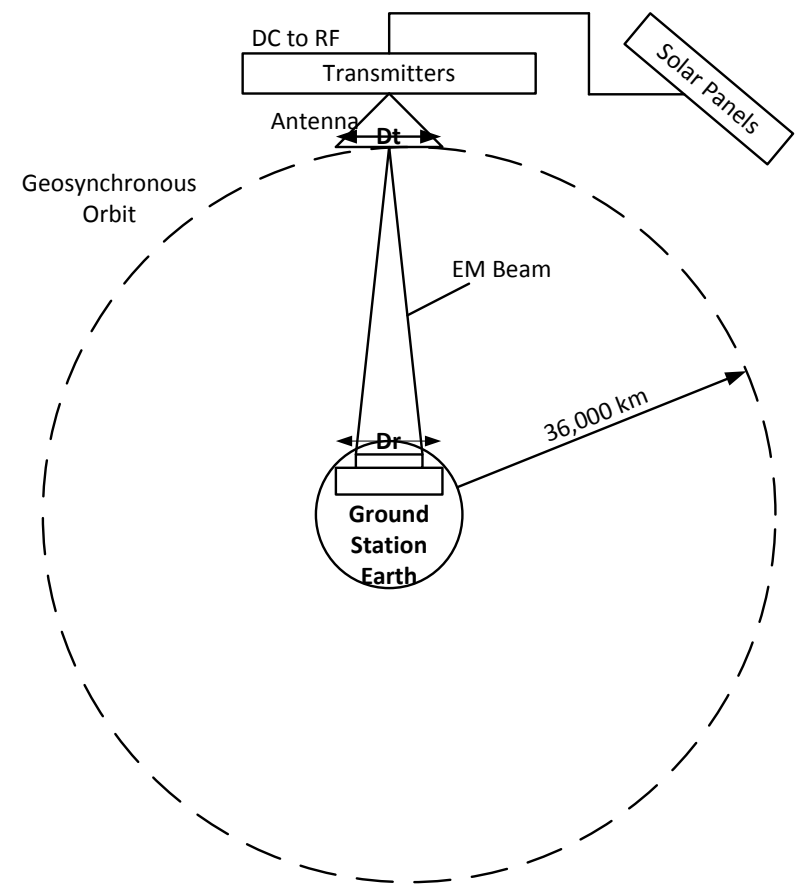

Figure 1 - Single Stage SSP-SS Architecture (typ.) Microwave frequency $5.8 \mathrm{GHz}$, GEO Transmit Antenna Diameter 1.3 km, Ground Rectenna Diameter 1.8 km.

via an electric cable from the tethered aerostat to a ground station, which is $95 \%$ - $99 \%$ efficient [8], though the full effects of corona at higher altitudes has not yet been fully accounted for. Corona losses can be reduced by selecting lower transmission voltages. Use of an electric cable largely avoids the effects of atmospheric attenuation that is concentrated in the region below $6 \mathrm{~km}$ above the earth's surface. The choice of the beaming frequency for the space-to-aerostat link would depend on various design parameters and trade-offs such as component availability, performance, efficiency, size, weight, and power levels. Other important factors are beam-pointing requirements, and antenna retro-directivity.

The second stage collection and rectification structure, converts incident EM power to DC electric power., The converter would be supported by a large aerostat tethered to the earth and provided with electric cables that would bring the rectified electric power directly to the earth. When microwave to high millimeter wave beaming frequencies are used, the converter is called a rectenna. In the case that optical frequencies are used for the power beam from space, band gaptuned photovoltaics would be used for conversion instead. In either case, an aerostat would host the high-altitude receiving portion. Aerostats are widely used items manufactured in the U.S. by companies such as ILC Dover, [9].

R. Dickinson has proposed using a powered stratospheric airship two-stage SPS system, [10]; in which the stratospheric airship at $20 \mathrm{~km}$ above the earth would support both a laser beam PV rectenna and a microwave transmitter/antenna, e.g. at $5.8 \mathrm{GHz}$. The microwave transmitter antenna aperture on the airship and the microwave rectenna area on the ground required by this system would be relatively small. However, this system would suffer from the inefficiencies inherent in requiring two rectenna stages, one on the airship and another

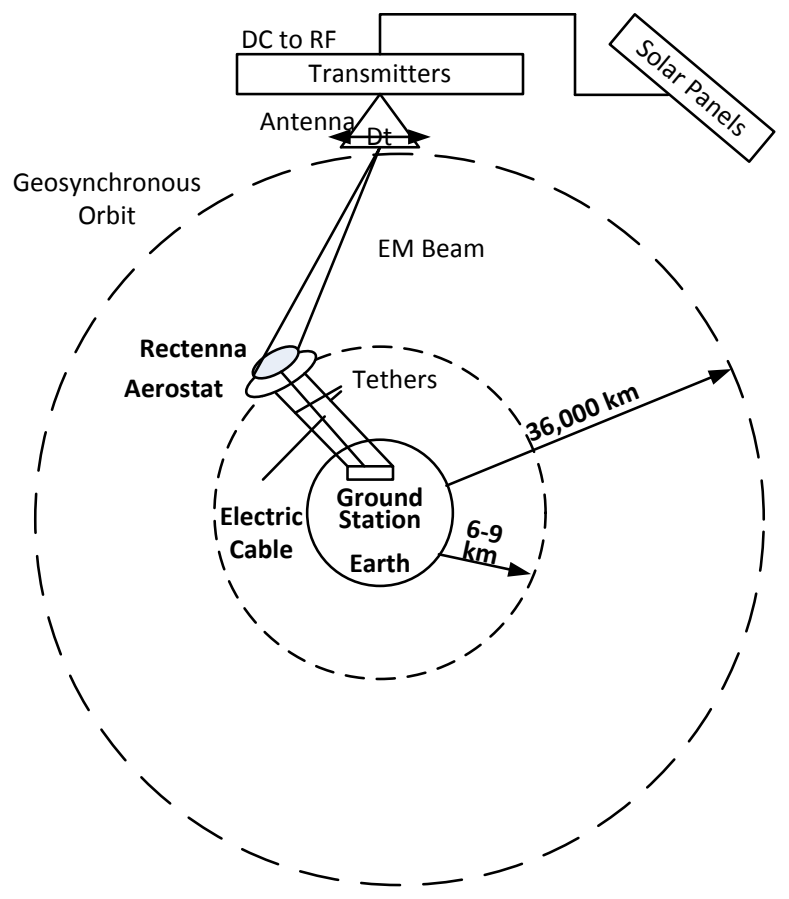

Figure 2 - Tethered Aerostat-SSP-TA-I Architecture (typ.), Laser wavelength 1.5 microns GEO Transmit Diameter 2.6 m, Tethered Aerostat Rectenna Diameter 40 m, Ground Station $\sim 50 \mathrm{~m} \times 50 \mathrm{~m}$

on the ground; and two DC-to RF conversions, one in space and another on the airship. The tethered aerostat architecture proposed here would have only one DC-to RF conversion (in space) and only one rectenna stage (on the aerostat).

The European Space Agency and Astrium/EADS, [11], have proposed a single-stage SPS system using laser beaming from GEO orbit in space directly to collection stations on the surface of the earth. A laser wavelength of $1.5 \mu \mathrm{m}$ was chosen by Astrium/EADS because this wavelength is purported to pose a minimal radiation hazard to humans or animals on earth at the power levels proposed. Astrium/EADS acknowledges that outages and sharp drops in power will occur due to variations in weather conditions in the lower atmosphere. Astrium/EADS also states that the development of a high power, space-ready laser at $1.5 \mu \mathrm{m}$ is the main technological challenge in the implementation of their system. At this 
wavelength laser diode efficiencies are currently approximately $40 \%$. The SSP-TA-I system proposed here, with its high altitude tethered aerostat, i) would not be subject to the effects of loss through the lower atmosphere, and ii) avoids the potential radiation hazard for personnel and equipment on the ground, thereby opening a wide range of possible beaming sources from $\mathrm{THz}$ to laser frequencies. The DARPA SHEDS program (Super High Efficiency Diode Sources) has demonstrated efficiencies greater than $70 \%$ at a wavelength of $940-975 \mathrm{~nm}$, [12]. High power $\mathrm{THz}$ sources are also being developed, [13].

2B. SSP-TA-I Advantages - The advantages of the proposed SSP-TA-I system are as follows:

1. The transmit aperture size required in space for efficient beaming would be proportionately smaller at the higher frequency SSP-TA-I system than at a lower frequency single stage system, thereby greatly reducing transportation costs to space. If a laser at 1.5 $\mu \mathrm{m}$ is used the transmit aperture diameter would be in the order of $2-3$ meters versus the kilometers of transmit aperture required by a lower frequency single stage system, e.g. $5.8 \mathrm{GHz}$, Figs. 1 and 2. The reduction in transportation costs to space would be in proportion to the reduction in size.

2. The ground station for the two-stage system is a relatively small structure, $50 \mathrm{~m} \times 50 \mathrm{~m}$ (typ.); and would be very much smaller than the kilometers of real estate required by a single stage system operating at a microwave frequency, e.g. 5.8 GHz.

3. The tethered aerostat architecture avoids the severe losses that can occur in energy transmission through the lower atmosphere due to variable weather conditions by continuously sending the received power down the tether; thereby providing a reliable, constant supply of electric power on a 24 hours per day, 365 days per year basis.

4. The receive aperture, that is supported by aerostats in the two-stage system, is designed using non-imaging concentrator techniques and is relatively small, much smaller than the kilometers of receive aperture that would be required by a single-stage SSP operating at a microwave frequency, e.g. $5.8 \mathrm{GHz}$. For power levels in the megawatt range, the receive aperture on the aerostat is approximately $40 \mathrm{~m}$ diameter. For higher powers, larger receive apertures and multiple aerostats may be required.

5. The energy beamed from space is focused onto the receive aperture 6-9 $\mathrm{km}$ above the earth. Any small amounts of spillover energy would be attenuated by the atmosphere before reaching earth, thereby turning atmospheric attenuation into an advantage. This would pre-empt the political/psychological fear factor of the potential radiation hazard for personnel and equipment that might otherwise prevent implementation of a space based solar power system.

6. The tethered aerostat architecture would have the capability to move the location of the ground station and aerostat. This could be done with relative ease as compared to a single stage low frequency system that would require kilometers of ground station collection area. The flexibility of ground station location would be of great importance when it is required to provide power to remote areas on an emergency basis, and of even greater importance in supplying power to military theaters of operation that are dynamic and rapidly changing.

7. Revenue produced by hosting telecommunication and observational payloads on the aerostat could significantly contribute to the financial viability of the system.

2C. SSP-TA-I DESIGN CHALLENGES-The main challenges in the design and implementation of the proposed SSP-TA-I system are:

1. Development of suitable, high power, efficient, reliable, space-qualifiable laser or $\mathrm{THz}$ sources.

2. Customization of existing aerostat designs to meet altitude, stability, endurance, and size requirements.

3. Increasing the efficiency of Solar to DC conversion and DC to RF generation in space, and of RF to DC rectification (or monochromatic PV conversion) on the tethered aerostat.

4. Meeting the difficult control and stabilization requirements for beam pointing and tracking from GEO orbit to the tethered aerostat. Techniques including the utilization of retro-directivity, pilot signals, diurnal motion sensing, will be investigated. Beam pointing and tracking in space orbit will require power.

5. Lowering of transportation costs to space.

6. The assembly in orbit of the power collection and transmission satellite.

7. To accommodate the need for continuous operation and limited life of the aerostat and its payload, a second complete aerostat system is needed per site to enable maintenance and repair on the ground.

8. The cable for power transmission from aerostat to ground will present a hazard to air travel, and needs to be incorporated into air-traffic management systems.

\section{CANDIDATE ARChITECTURE SSP-TA-II}

3A. Description-We now discuss the system architecture shown in Figure 3 designated by SSP-TA-II. In this proposed system, there is no down conversion (rectification) of solar energy to DC electric energy and then an up conversion from DC to some high frequency RF transmitter required to be done in GEO orbit in space. Instead, a uniquely designed optical system for the collection and direct transmission of solar energy from space to the tethered aerostat is proposed. The potential advantages of this architecture in terms of increased system efficiency and reliability and reduced cost are great. It must be stated, however, that this approach requires a major $\mathrm{R} \& \mathrm{D}$ advance in optical system design involving recent developments in non-imaging optics, reflector concentrator and fiber cable design; whereas the SSP-TA-I architecture, Figure 2, described above, requires an evolutionary R\&D effort in existing concepts and hardware. 


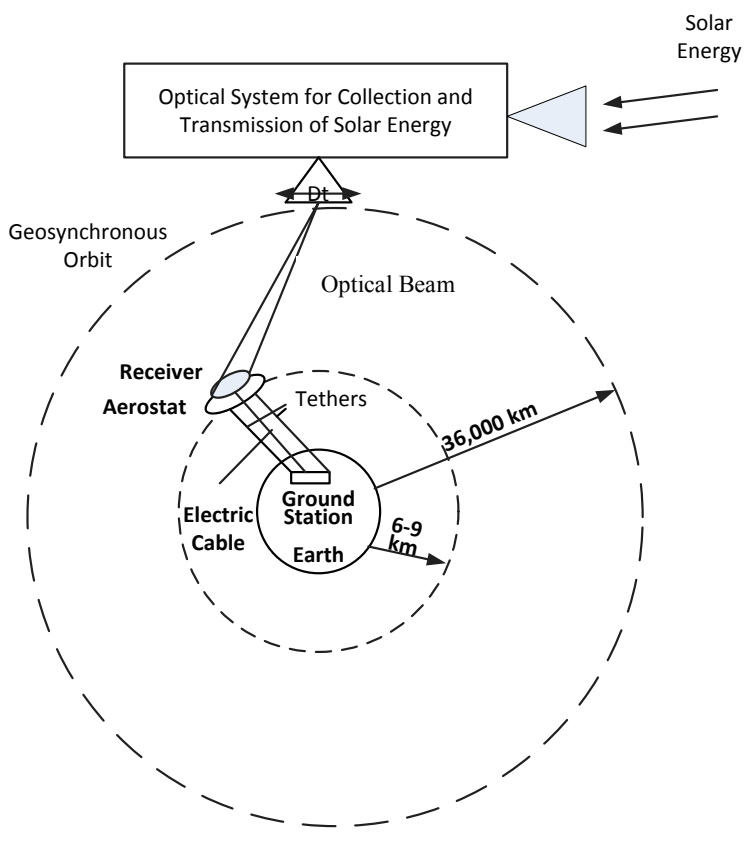

Figure 3 - Tethered Aerostat SSP-TA-II Architecture, Tethered Aerostat Rectenna Diameter 40m, Ground Station 50m x 50m

The main challenge to the successful implementation of the SSP-TA-II architecture is the design of an optical system for the efficient collection and direct transmission of solar energy from space to the tethered aerostat. Natural sunlight is not fully collimated and has a divergence angle of approximately 0.01 radians. In addition, the energy in sunlight is distributed over a broad spectrum of frequencies.

In order to achieve the advantages of the SSP-TA-II architecture an optical system must be designed that operates over a sufficiently broad range of frequencies and that reduces the divergence angle of an appropriately sized beam of sunlight to approximately $10^{-6}$ radians. To do this for a single radiating aperture is a difficult task. However, such performance might be effectively obtained by using an array of offset radiating apertures each with a divergence angle of $10^{-4}$ $-10^{-5}$ radians; and each beam having a specified overlap at the target receive aperture. The optical processing must minimize loss, i.e. minimize any increase in system etendue, while allowing increased beam area and decreased divergence. Possible techniques for achieving this are: i) An array of single mode optical waveguides with specially designed input and output lenses, ii) Inverse cassegrain reflectors with shaped sub reflectors, and iii) Directional coupling of sunlight to selected materials that exhibit stimulated emission. These techniques and others can be investigated as a means to enable the beaming of sunlight from GEO to $6 \mathrm{~km}$ above the earth with a spot size diameter of approximately $50 \mathrm{~m}$. We emphasize that we are reporting on a preliminary feasibility study and not a design study.

3B. Advantages of the Proposed SSP-TA-II System-The SSPTA-II system has essentially all the previously enumerated advantages of the SSP-TA-I system plus the following:

1. Greatly improved efficiency in delivering usable electric power from space to earth by avoiding inherently inefficient intermediate energy conversions. The conventional single stage system requires multiple energy conversions: solar to dc to microwaves in space and microwaves to $\mathrm{dc}$ on the ground. The SSP-TA-II architecture proposed here would have only one energy conversion: solar to DC on the tethered aerostat.

2. The prospect of reduced transportation costs to space. The conventional single stage system requires an array of solar panels and a very large RF transmitting aperture in space orbit. The SSP-TA-I also requires an array of solar panels in space orbit. The SSP-TA-II architecture would have solar panels only on the tethered aerostat and has no need for an array of solar panels and a large RF transmitting aperture in orbit in space.

3. The optical system in GEO orbit for the collection and transmission of solar energy would be comprised of passive optical components and would not require active amplification, thereby greatly increasing its reliability.

\section{C. SSP-TA-II Design Challenges-}

1. The main challenge in the design and implementation of the SSP-TA-II system is the development of an efficient optical system for the collection and direct transmission of solar energy from space to the tethered aerostat. This approach requires a state-ofthe-art R\&D advance in optical system design involving recent developments in non-imaging optics, reflector concentrator, optical waveguide and fiber cable design.

2. Customization of existing aerostat design to meet altitude, stability, endurance and size requirements.

3. Increasing the efficiency of sunlight to DC conversion via utilization of non-imaging concentrator design.

4. Meeting the difficult control and stabilization requirements for beam pointing and tracking from GEO orbit to the tethered aerostat. Techniques including the utilization of retro-directivity, pilot signals, diurnal motion sensing, will be investigated. Beam pointing and tracking in space orbit will require power.

5. To accommodate the need for continuous operation and limited life of the aerostat and its payload, a second complete aerostat system is needed per site to enable maintenance and repair on the ground.

6. The cable for power transmission from aerostat to ground will present a hazard to air travel, and needs to be incorporated into air-traffic management systems.

\section{PoWered Airship Alternative to the TETHERED AEROSTAT}

The SSP-TA system architecture discussed in this proposal features a tethered aerostat being used to place an aperture in vicinity of 6 or $9 \mathrm{~km}$ altitude. In this region, wind can be a significant factor affecting the aerostat. For this reason, we will also consider an alternate platform; a powered airship (PA), 
[14]. This alternative system architecture is designated SSPPA. The recent flight of the Long Endurance Multi-intelligence Vehicle (LEMV) suggests that a powered airship lifting body could be a better solution to placement of the aperture. In addition to its large size, LEMV has the capability to lift 2500 lbs to the $6 \mathrm{~km}$ altitude while carrying a propulsion system that could be used to provide stability. Instead of the traditional aerostat tether which provides both mechanical restraint and an electric power cable, we envision a lighter cable dedicated to electric power transmission. The size and weight of the cable/tether and the attenuation of electric power in transmission to the ground are important technical issues for either aerostat or airship platforms, [15]. The electric cable diameter size is largely dictated by the power required to be transmitted. The weight and electric loss can be minimized by the use of high transmission voltage and low resistivity conductors. ILC Dover manufactured the envelope for LEMV under contract to Northrop Grumman. The above discussion of the SSP-TA-I and SSP-TA-II system advantages and challenges would carry over to SSP-PA-I and SSP-PA-II systems, with the powered airship possibly providing better aerodynamics and station keeping performance.

\section{CoMparison OF THE ARCHITECTURES}

Estimates of subsystem dimensions and total system efficiencies of the three system architectures discussed above are shown in Table 1. This comparison is based on very preliminary estimates of performance. For purposes of the comparison, the distance from GEO to ground is taken to be $36,000 \mathrm{~km}$, and it is assumed that $1 \mathrm{MW}$ of solar energy is collected in GEO by each of the systems. It is also assumed that the beaming frequency for the SSP-SS system is 2.45 $\mathrm{GHz}$, for the SSP-TA-I system the beaming frequency is 158 THz and that the SSP-TA-II beams sunlight.

\section{Table 1. Estimated Subsystem Dimensions and} Performance

\begin{tabular}{|l|l|l|l|}
\hline & SSP-SS & SSP-TA-I & SSP-TA-II \\
\hline $\begin{array}{l}\text { Photovoltaic panels } \\
\text { in GEO Diameter } \\
(\mathrm{m})\end{array}$ & $55^{*}$ & $55^{*}$ & 0 \\
\hline $\begin{array}{l}\text { Solar reflector } \\
\text { panels in GEO } \\
\text { Diameter (m) }\end{array}$ & $60^{*}$ & $60^{*}$ & $60^{*}$ \\
\hline $\begin{array}{l}\text { RF/optical GEO } \\
\text { transmit diameter } \\
\text { (m) }\end{array}$ & 2,600 & 5 & 10 \\
\hline $\begin{array}{l}\text { Solar rectifier } \\
\text { panels in upper } \\
\text { troposphere, } \\
\text { Diameter (m) }\end{array}$ & 0 & 40 & 50 \\
\hline $\begin{array}{l}\text { Ground station, } \\
\text { Diameter (m) }\end{array}$ & 4,000 & 50 & 50 \\
\hline $\begin{array}{l}\text { Power delivered } \\
\text { (KW) }\end{array}$ & 97 & 59.5 & 152 \\
\hline
\end{tabular}

*1 MW assumed to be collected in GEO. More power would require larger collection and rectifying apertures.

**The volume and mass of RF/optical transmitter power modules in GEO is not included in the above estimates. For the SSP-SS, it would be extremely large; for the SSP-TA-I, it would be substantial; but for the SSP-TA-II it would be zero.
From the data shown in Table 2, with system efficiency, $\eta$ tot, defined as the ratio of Power Delivered to Solar PowerIn, we obtain the following:

SSP-SS, $\eta$ tot $=9.7 \%$; SSP-TA-I, $\eta$ tot $=6 \%$; SSP-TA-II, $\eta$ tot $=$ $15 \%$.

Table 2. Efficiencies

\begin{tabular}{|l|c|c|c|}
\hline $\begin{array}{l}\text { Efficiency } \\
\text { per stage }\end{array}$ & SSP-SS & SSP-I & SSP-II \\
\hline $\begin{array}{l}\text { PV } \\
\text { conversion }\end{array}$ & $30 \%$ & $30 \%$ & $30 \%$ \\
\hline $\begin{array}{l}\text { DC to RF } \\
\text { conversion }\end{array}$ & $60 \%$ & $40 \%$ & ----- \\
\hline Beaming & $90 \%$ & $90 \%$ & $90 \%$ \\
\hline $\begin{array}{l}\text { RF to DC } \\
\text { conversion }\end{array}$ & $70 \%$ & $70 \%$ & $60 \%$ \\
\hline DC cable & $90 \%$ & $90 \%$ & $90 \%$ \\
\hline $\begin{array}{l}\text { Total } \\
\text { efficiency }\end{array}$ & $10 \%$ & $6 \%$ & $15 \%$ \\
\hline
\end{tabular}

At this early stage of our investigation, it must be stated that these efficiency numbers are based on very preliminary estimates and should be understood to give qualitative estimates rather than accurate quantitative values. For example, in the literature there are widely divergent estimates of photovoltaic conversion efficiencies, ranging from $12 \%$ to $70 \%$ [3], [7], [9]. The relatively conservative values of efficiencies for each stage shown in Table 2 are used to obtain qualitative estimates of total power-out-to-power-in system efficiency. But power-out-to-power-in efficiency is hardly the entire story given the many additional objective and subjective factors of cost, performance, politics, commercial interests and public psychology.

The above proviso about the preliminary nature of our investigation notwithstanding, it is clear that the difference in the amount of hardware in space and on the ground between the single-stage SSP-SS system and either the SSP-TA-I or the SSP-TA-II system is significant. Accordingly, the difference in cost for transportation to space and for real estate and maintenance on the ground, between the SSP-SS system and either of the two SSP-TA systems, would also be significant [5], [6]. This cost differential would be only minimally offset by the cost of a tethered aerostat or powered airship required by the SSP-TA systems.

The beaming frequency of $158 \mathrm{THz}$ (1.90 microns) used in the above table for the SSP-TA-I system would propagate from GEO to the top of the troposphere with little loss, but would be almost completely absorbed in the lower atmosphere before it could reach the ground. This would effectively eliminate the potential radiation hazard to personnel and equipment on the ground. Many other beaming frequencies having similar properties are available, Figure 4. 


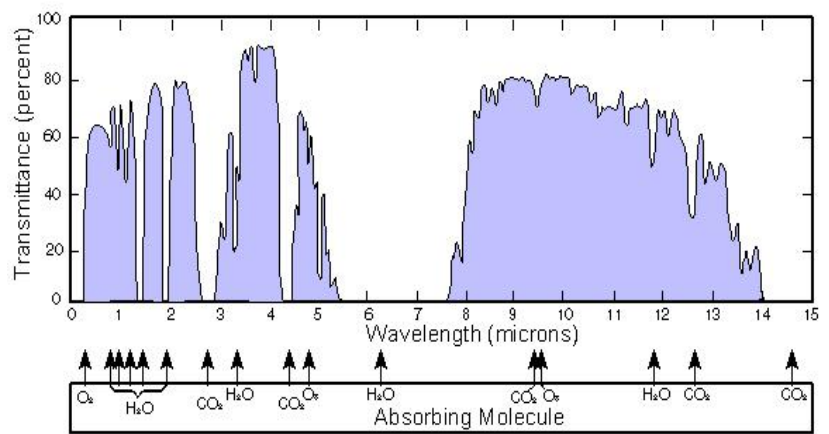

Figure 4 - Atmospheric Transmittance vs. Wavelength

\section{Conclusions}

A preliminary, unfunded feasibility study of two-stage Space Based Solar Power to Tethered Aerostat to Earth (SSP-TA) system architectures has been presented. This study does not purport to provide quantitative, definitive conclusions about the feasibility of either of the two-stage SSP-TA architectures discussed, nor of the referenced single-stage SSP architecture. More definitive conclusions would result from the execution of a rigorous, analytic, experimental, and properly funded study. A simplified estimated ranking of the three systems in terms of specific performance parameters is given in Table 3 .

It is clear to these investigators, that space-based solar power can provide a significant breakthrough in solving our national energy requirements both on a commercial utility level and for defense and national security needs, if its critical challenges can be adequately addressed. It is likely that space solar power technology development and demonstration efforts would yield a myriad of secondary and tertiary benefits for entities that pursue them, even if the concept does not come to fruition in the near term.

Table 3. Comparative System Performance Ranking

\begin{tabular}{|l|c|c|c|}
\hline & \multicolumn{3}{|c|}{ System } \\
\hline $\begin{array}{l}\text { Performance } \\
\text { Parameter }\end{array}$ & SSP-SS & SSP-TA-I & SSP-TA-II \\
\hline Cost & Very high & High & Least costly \\
\hline Ground Station Area & Very large & Small & Small \\
\hline Radiation Hazard & $\begin{array}{c}\text { Moderately } \\
\text { high }\end{array}$ & Very low & Very low \\
\hline Power supply reliability & Good & Good & Good \\
\hline Hardware reliability & Relatively high & $\begin{array}{c}\text { Relatively } \\
\text { low }\end{array}$ & $\begin{array}{c}\text { Relatively } \\
\text { high }\end{array}$ \\
\hline
\end{tabular}

\section{REFERENCES}

[1] Method and apparatus for converting solar radiation to electrical power, by P. E. Glaser (1973). U.S. Patent 3781 647. Available: http://www. freepatentsonline.com/3781647.pdf

[2] W.C. Brown. (1979, Jun.). Solar power satellites: microwaves deliver the power. IEEE Spectr.16(6), pp. 36-42.

[3] J. Mankins. (2012, Sept. 15). SPS-ALPHA: The first practical solar power satellite via arbitrarily large phased array (a 2011-2012 NASA NIAC phase 1 project). Available: http://www.nasa.gov/pdf/716070 main_Mankins_2011_PhI_SPS_Alpha.pdf

[4] A. Globus. (2009, Jan.). In defense of space solar power [Online]. Available: http://space.alglobus.net/papers/ FetterResponse.html

[5] Space based solar power to aerostat system, Provisional Patent by S. J. Blank (2012, Aug. 6).
[6] S.J. Blank (2012, May 28). Space solar power to tethered aerostat to ground. Presented at ISDC 2012 Conf, NSS, Wash. DC.

[7] V. K. Sysoev, V. Khartov, K. M. Pichhadze, A.A. Verlan, A. D. Ponomarenko. New solutions with regard to space solar energy will bring closer theory and practice. Presented at $63^{\text {rd }}$ International Astronautical Congress, Naples, Italy, Oct. 4, 2012.

[8] American Electric Power. Transmission Facts. Available: http://www.aep.com/about/transmission/docs/transmission-facts.pdf

[9] ILC Dover. Lighter-than-Air Structures. Available: http://www.ilc dover. com/Lighter-Than-Air-Structures/ (2012).

[10] Dickinson, R. M.,(2005). "A concept GEO-SPS \& airship multimegawatt power relay system," Journal of the British Interplanetary Society, Vol. 58, No.9/10, pp. 298-301, Sept./Oct. 2005.

[11] M. Perren, Astrium, private correspondence.

[12] M. Peters, V. Rossin, M. Everett, E. Zucker, High Power, High Efficiency Laser Diodes at JDSU. Available: http://www.jdsu.com/ ProductLiterature/PW07_JDSU_6456_13.pdf

[13] B.S. Williams, S. Kumar, Q. Hū, J.L. Reno. (2006, Jan 19). High-power terahertz quantum-cascade lasers. Electronic Letters 42(2). Available: http://www.rle.mit.edu/thz/ documents/01582070.pdf.

[14] T. Miller, M. Mandel. (2000, Jul. 5). Airship envelopes: requirements, materials, and test methods. Presented at $3^{\text {rd }}$ Biennial Inter'l Airship Convention.

[15] TCOM, L.P, Platforms. Available: http://www.tcomlp.com/platforms/ (2013)

\section{Biography}

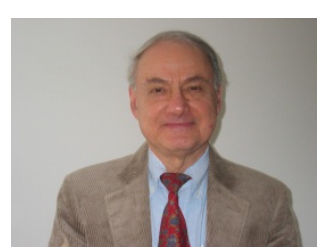

Stephen J. Blank, Ph.D., has worked on research and development in the field of antennas, microwaves and electromagnetics for many years. At AIL, L.I., NY, he worked on antenna $R \& D$. At the Grumman Aerospace Corp, L.I., NY, he was Antenna Group Leader on the F-111B project and co-inventor of the circularly polarized trihedral reflector, used for automated carrier landings by the U.S. Navy, U. S. patent 3,309,705. In 1984, he received a NASA Certificate of Recognition for developing the algorithm for the Empirical Optimization of Antenna Arrays. At JPL, Pasadena, CA, he worked on the design of array feeds for distortion correction and vernier beamsteering of large reflector antennas. In 1982 he founded a consulting company, IBE Systems; working mainly in the field of broadcast engineering. He co-authored the chapter on Directional Couplers for the Handbook of Microwave Technology, Academic Press, 1995. He has worked on the development of array antennas for MRI systems and has had various papers published in the IEEE Transactions on Antennas and Propagation. He was technical chairman of the Task Force on Energy Conservation and Radiation Hazard Reduction, IEEE LI section. In 1995, he received the IEEE Region 1 Electrical Engineering Professionalism Award for his contributions to energy conservation and radiation hazard reduction. In 1998, he was invited to lecture on the subject of Vertical Polarization for FM Broadcasting at the 21st Congress of the Association of Brazilian Broadcasters in Iguacu, Brazil. At NYIT, he conducted research funded by the U.S. Army on the use of microwave and millimeter wave radiation for wireless power transmission. He presented a paper on Space Based Solar Power at the International Space Development Conference, Wash. D.C., May 28, 2012; and a paper on Wireless Power Transmission at IEEE MTT Symposium, Montreal Canada, on June 18, 2012. He is a Professor of Electrical and Computer Engineering at the New York Institute of Technology, Old Westbury, NY. He is currently conducting research on space based solar power. 


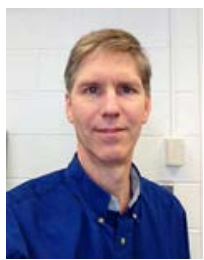

Stephen J. Leete., is a systems engineer at the NASA Goddard Space Flight Center since 1987, currently in the Mission Systems Engineering Branch. He has worked on several space flight missions, including the Cosmic Background Explorer, Cassini Composite Infrared Spectrometer, Landsat 7 , three Hubble Space Telescope servicing missions, Constellation, and the Astrophysics Projects Division. He worked for Hughes Aircraft Company on Intelsat VI. He received his BSE from Princeton University in 1981, where his senior independent work advisor was Gerard K. O'Neill, and received his MSE from George Washington University in 1997.

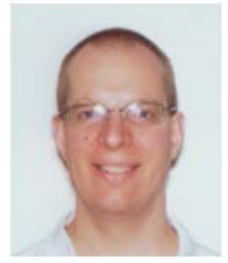

Paul Jaffe is an electronics engineer, researcher, and integration and testing section head at the Naval Research Laboratory. He has worked on over a dozen NASA and Department of Defense space missions, including SSULI, STEREO, TacSat1. TacSat-4, and MIS. He developed standards and lead spacecraft computer hardware development as part of the Department of Defense's Operationally Responsive Space effort. He served as a coordinator and editor of NRL's Space Solar Power (SSP) study report and is presently the principal investigator for an SSP-related research effort. His current primary role is as the Electrical Segment Lead for the Weather Satellite Follow-on microwave sensor effort. Paul is also active in educational and STEM outreach. He has a B. S. from the University of Maryland and a M. S. from the Johns Hopkins University, both in Electrical Engineering. He is currently pursuing a Ph.D. at the University of Maryland.

\section{Acknowledgements}

The authors are grateful for the help given in preparation of this paper by Phil Spampinato and Steve Scarborough, ILC Dover. 